\title{
Bibliometric analysis of publication activity in the field of familial Mediterranean fever in 2010-2019: a Scopus-based study
}

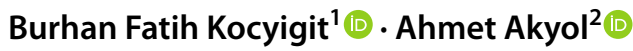

Received: 20 June 2021 / Accepted: 2 September 2021 / Published online: 9 September 2021

(c) The Author(s), under exclusive licence to Springer-Verlag GmbH Germany, part of Springer Nature 2021

\begin{abstract}
Familial Mediterranean fever (FMF) is a systemic autoinflammatory disease, which presents with recurrent episodes of fever, serositis, synovial inflammation and various different clinical features. In parallel with the geographical distribution of FMF, the prevalence is notably higher in some population groups including Turks, Jews, Armenians and Arabs. The aim of this study was to assess FMF articles published between 2010 and 2019 bibliometrically and to present up-to-date data. This bibliometric research was conducted on the Scopus database. The key term "familial Mediterranean fever" was utilized to generate the publication list. The number of articles, active countries journals, prolific authors and citation data were determined. Visualization maps that revealed collaboration networks were also created. The number of FMF articles displayed a stable trend between 2010 and $2019(p>0.05)$. The top five countries according to the number of articles were Turkey $(n=473 ; 52.90 \%)$, Israel $(n=86 ; 9.61 \%)$, Japan $(n=39 ; 4.36 \%)$, Italy $(n=38 ; 4.25 \%)$ and the USA $(n=37 ; 4.13 \%$. Italy, the USA and France were ranked as the top three countries for the average number of citations per article. Rheumatology International, Clinical Rheumatology, Clinical and Experimental Rheumatology, Annals of Rheumatic Diseases and Pediatric Rheumatology were the top five journals according to the number of FMF articles. The geographical distribution of FMF was seen to affect the article production of countries. Although Turkey dominated the field in the number of articles, it was not at the forefront in the average number of citations. To increase the production of articles in the field of FMF, it is necessary to support researchers in countries other than those which are already strongly active.
\end{abstract}

Keywords Familial Mediterranean fever $\cdot$ Bibliometric analysis $\cdot$ Scopus $\cdot$ Publication trend $\cdot$ FMF

\section{Introduction}

Familial Mediterranean fever (FMF) is the cornerstone disease of the systemic autoinflammatory disorders group and presents with recurrent fever attacks, serositis that can occur in different regions, synovial inflammation and various different clinical features [1,2]. There is a high prevalence in the Mediterranean region indicating a distinct geographical distribution and, consequently, Turkish, non-Ashkenazi

Burhan Fatih Kocyigit

bfk2701@hotmail.com

Ahmet Akyol

ahmetakyol07@gmail.com

1 Faculty of Medicine, Department of Physical Medicine and Rehabilitation, Kahramanmaraş Sütçü İmam University, Kahramanmaraş, Turkey

2 Physiotherapy and Rehabilitation Application and Research Center, Hasan Kalyoncu University, Gaziantep, Turkey
Jewish, Armenian and Arab populations frequently encounter the disease. However, FMF cases also emerge in different regions outside of this geographical distribution [3-5]. As a result of various mutations, a defect occurs in the pyrin/ marenostrin-related MEFV gene, which is followed by activation of caspase- 1 and increased secretion of interleukin-1, which is the key cytokine of inflammation in FMF [6, 7].

Due to the ever-expanding literature, it is quite difficult for researchers to have knowledge about all the publications on a particular topic, and so bibliometric studies have become increasingly important. Bibliometric analysis is accepted as a reliable method for measuring scientific productivity, revealing trend analyses and identifying productive countries and authors by focusing on the number of articles and citations published on a particular subject $[8,9]$. Another point emphasized in bibliometric studies is the collaborations between countries, authors and institutions in scientific production [10]. Researchers can rapidly reach comprehensive information about a particular subject, 
determine the trends and hot topics in recent years and plan their future research accordingly through the data provided from bibliometric analyses [11].

Two bibliometric studies have been previously conducted on FMF [6, 12]. While one of the studies focused on articles from Arab countries [6], the other examined the top-100 cited articles [12]. The aim of the current study was to globally evaluate 10 years (2010-2019) of FMF articles through the Scopus database and thereby provide data on the global publication trend, influential authors, dominant countries and journals. In addition, it was aimed to clarify the global network by creating visualization maps.

\section{Materials and methods}

In this bibliometric study, the Scopus database was used to list the articles published in the field of FMF on May 30, 2021. There are various reasons for choosing the Scopus database. Compared to databases such as Web of Science and Pubmed, Scopus presents a wider journal coverage. Scopus covers the entire Medline and includes more than 23,000 indexed journals from medical and other scientific disciplines [13]. The citation data available from Scopus is comprehensive, and the data presented by Scopus can be readily transferred to Microsoft Excel or VOSviewer software with a few simple steps for bibliometric analysis and visualization [14]. Therefore, Scopus is a frequently referred database in bibliometric analyses and has reached a high level of reputation in this field [15-17].

Title and/or abstract (TITLE-ABS) search was selected and the key term "familial Mediterranean fever" was used in the creation of the publication list $[18,19]$. The article listing strategy was limited to 'title or abstract' to obtain more accurate search results, as it was noticed that if the search strategy was expanded with different search options such as keywords, a large number of publications not related to FMF were listed. It has been noted that the narrowed search strategy leads to a significant increase in specificity with minimal reduction in sensitivity compared to topic search (title-abstract-keywords) [20, 21]. The date range was restricted to January 2010 to December 2019 and only publications in English were included. The publication type was selected as original article and review. Other types of publications were excluded. Following these steps, a saved list was created on Scopus and two researchers (BFK and AA) reviewed the titles and abstracts to determine whether the articles were directly related to FMF. When doubt arose about an article, its full text was accessed and the final decision was made. Duplicate papers were identified and only one was kept in the list. After all these processes, the remaining article data were transferred to Microsoft Excel 2016 for Windows (CSV format) and was also recorded in RIS format.

The bibliometric determinants used to analyze FMFrelated articles were total number of articles, year of article, country of article, journal, author and citation data. In studies with authors from multiple countries, the country of the corresponding author was recorded as the country of the article [22, 23]. Funding sources and authors' h index data were obtained from Scopus. From 2010 to 2019, the number of articles produced each year was calculated. The criterion for classification as a main active country was defined as 'producing $1 \%$ or more of the total number of articles published from 2010 to 2019 [24, 25]. The population size and gross domestic product (GDP) data of the main active countries were obtained from the 'https://www.cia.gov/theworld-factbook/countries/' website. The average number of citations value was calculated with the formula of 'the total number of citations $\div$ the total number of articles'. The five most prolific authors, the five most active journals and top five funding sources were also determined.

The most frequently used author keywords were obtained from the VOSviewer software (version 1.6.16). The data obtained from Scopus were easily processed in this software and visualization mapping was performed. Visualizations were created with the analyses of country co-authorship, author co-authorship, cited source co-citation and author keyword co-occurrence. In the VOSviewer, the visualization maps are created by combining countries, authors, journals or keywords that have a certain number of links. The thickness of the lines between items expresses the relative strength of the connection between them, with a thicker line representing a stronger connection. In addition, separate clusters are formed and presented in similar colors [26].

\section{Statistical analysis}

Data obtained in the study were analyzed statistically using the Statistical Package for the Social Sciences version 20.0 software (SPSS Inc., Chicago, IL, USA). The results of the bibliometric analyses were expressed as number $(n)$ and percentage (\%). The trend analysis based on the number of articles between 2010 and 2019 was revealed using regression analysis. The statistical significance level was accepted as 0.05 .

\section{Results}

In the first phase, a total of 3948 FMF publications were listed without any restrictions. After the whole inclusion-exclusion process, a total of 894 articles were obtained for bibliometric analyses (Fig. 1). Articles were provided from 44 different countries and 13 of these met the main 


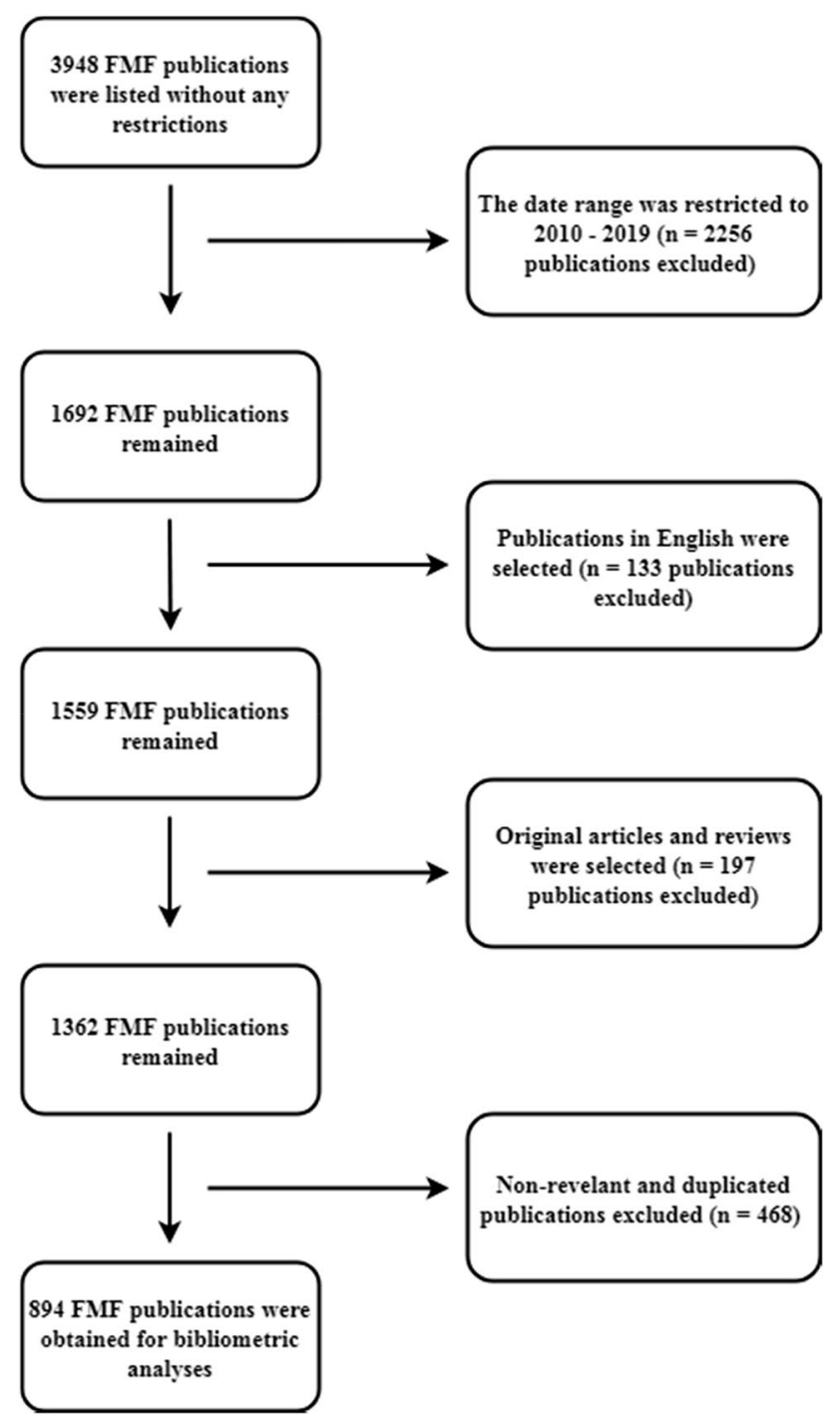

Fig. 1 Flowchart revealing the selection of FMF publications

active country criteria. The main active countries produced $91.95 \%(n=824)$ of the articles. When the distribution of the number of articles by years was investigated, the value was 68 in 2010 and 85 in 2019 (Fig. 2). Trend analysis was applied based on the number of articles published each year from 2010 to 2019, and a stable course was determined $(p>0.05)$. No significant trend was detected indicating an increase or decrease.

The top five countries according to number of articles were Turkey $(n=473 ; 52.90 \%)$, Israel $(n=86 ; 9.61 \%)$, Japan $(n=39 ; 4.36 \%)$, Italy $(n=38 ; 4.25 \%)$ and the USA $(n=37$; $4.13 \%$ ), respectively (Table 1 ). A country co-authorship visualization map representing international collaborations was created. Articles with authors from more than 25 different countries were omitted and the full counting method was selected. The minimum number of articles and citations of a country was set to 8 and 50, respectively. These criteria were

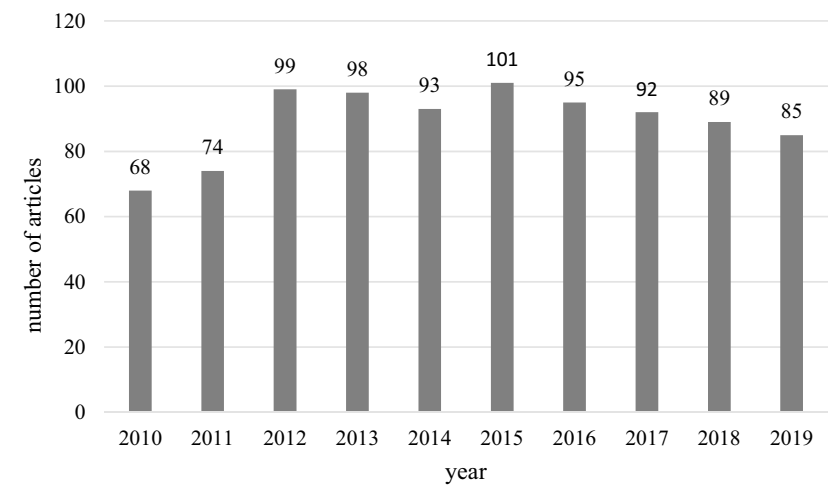

Fig. 2 Publication trend from 2010 to 2019

met by 17 countries. Weighting was performed according to the number of publications, and the circle size on the map represents the number of articles (Fig. 3).

In the calculations performed according to the population of the countries, Israel (9.79), Armenia (5.98) and Turkey (5.73) were ranked in the first three places for the number of articles per million population. In the GDP-adjusted article count data, Armenia (45), Israel (23.88) and Turkey (19.95) were the top three. The three leading countries for the number of citations per article were Italy (36.41), the USA (28.51) and France (27.31) (Table 1).

The five most prolific authors were Ozen $\mathrm{S}(n=53$; 5.92\%; from Turkey), Livneh A ( $n=51 ; 5.70 \%$; from Israel), Lidar M ( $n=27 ; 3.02 \%$; from Israel), Ben-Zvi I ( $n=26$; $2.90 \%$; from Israel) and Cantarini L $(n=23 ; 2.57 \%$; from Italy), as presented in Table 2 . The author co-authorship

Table 1 The main active countries between 2010 and 2019

\begin{tabular}{lccccc}
\hline Country & $n(\%)$ & $n^{\mathrm{a}}$ & \multicolumn{1}{l}{$n^{\mathrm{b}}$} & Total citations & $\begin{array}{l}\text { Average } \\
\text { citations }\end{array}$ \\
\hline Turkey & $473(52.90)$ & 5.73 & 19.95 & 5248 & 11.09 \\
Israel & $86(9.61)$ & 9.79 & 23.88 & 1454 & 16.90 \\
Japan & $39(4.36)$ & 0.31 & 0.74 & 495 & 12.69 \\
Italy & $38(4.25)$ & 0.61 & 1.48 & 1384 & 36.42 \\
USA & $37(4.13)$ & 0.11 & 0.18 & 1055 & 28.51 \\
France & $29(3.24)$ & 0.42 & 0.93 & 792 & 27.31 \\
Iran & $25(2.79)$ & 0.29 & 2.45 & 150 & 6.00 \\
Egypt & $25(2.79)$ & 0.23 & 2.11 & 134 & 5.36 \\
Germany & $18(2.01)$ & 0.22 & 0.40 & 280 & 15.55 \\
Armenia & $18(2.01)$ & 5.98 & 45 & 211 & 11.72 \\
Greece & $16(1.78)$ & 1.51 & 5.16 & 211 & 13.18 \\
UK & $9(1.01)$ & 0.13 & 0.28 & 195 & 21.66 \\
Lebanon & $9(1.01)$ & 1.71 & 10 & 57 & 6.33
\end{tabular}

$n$ : number of articles, $\%$ : percentage

$n^{\mathrm{a}}$ : number of articles per million population, $n^{\mathrm{b}}$ : number of articles per $\$ 100$ billion gross domestic product 


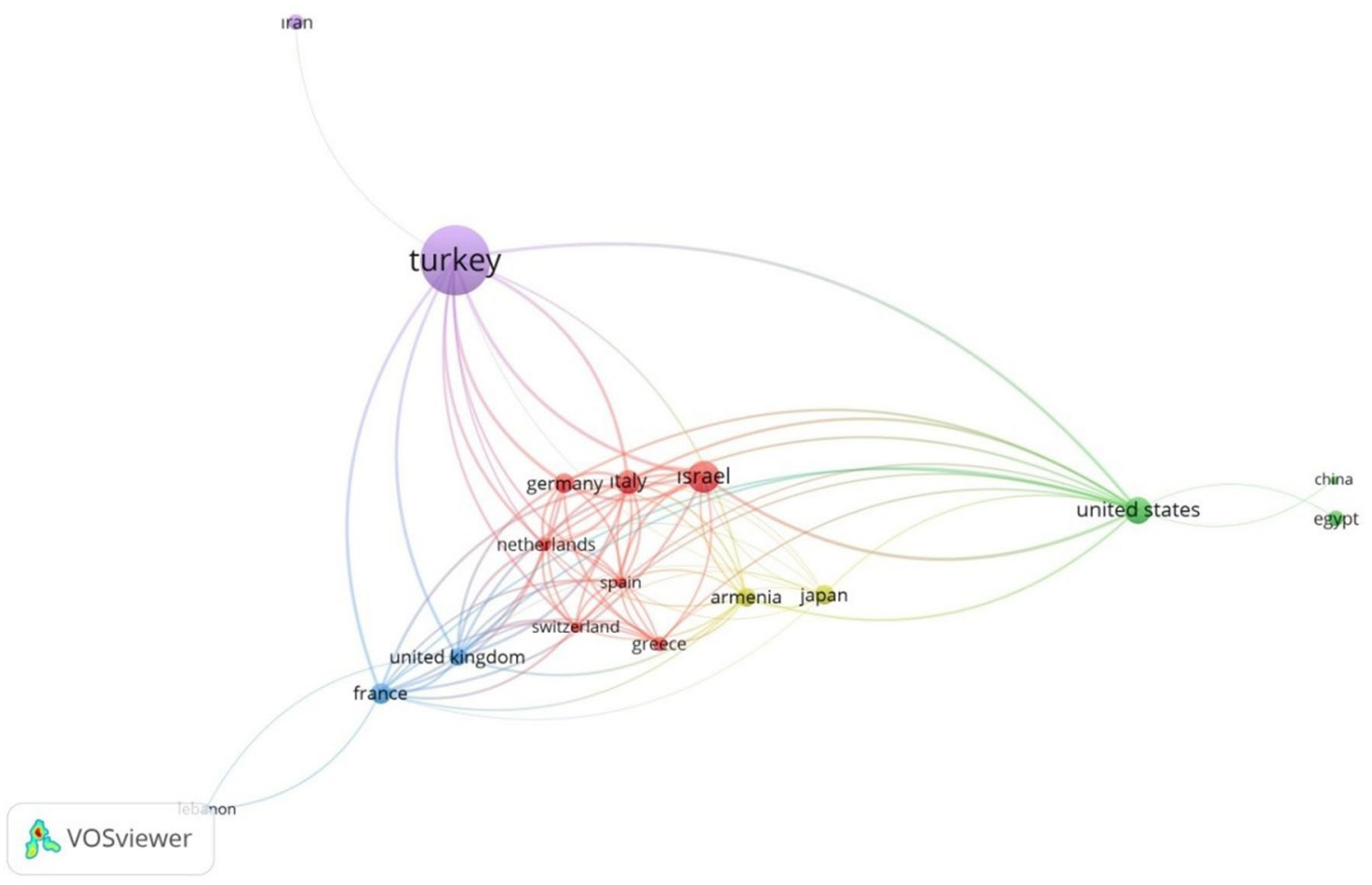

Fig. 3 Country co-authorship network visualization map. Five clusters were created. Cluster 1 (Germany, Greece, Israel, Italy, Netherlands, Switzerland, Spain) is presented in red; Cluster 2 (China,

Table 2 Top five most prolific authors

\begin{tabular}{llrll}
\hline Author & $n(\%)$ & Total citations & Average citations & $\begin{array}{l}\text { Scopus } \\
h \text { index }\end{array}$ \\
\hline Ozen S & $53(5.92)$ & 1905 & 35.94 & 71 \\
Livneh A & $51(5.70)$ & 1224 & 24 & 55 \\
Lidar M & $27(3.02)$ & 470 & 17.40 & 31 \\
Ben-Zvi I & $26(2.90)$ & 513 & 19.73 & 15 \\
Cantarini L & $23(2.57)$ & 1199 & 52.13 & 49 \\
\hline
\end{tabular}

$n$ : number of articles, $\%$ : percentage

network visualization map is shown in Fig. 4. Prior to visualization, an author's minimum number of articles and citations were set to 10 and 50, respectively, which resulted in 36 authors. Visualization was carried out according to the number of articles produced.

Journals were ranked according to the number of articles they published in the FMF field. The ranking was as follows: Rheumatology International (Rheumatol Int) $(n=50$; $5.59 \%)$, Clinical Rheumatology (Clin Rheumatol) $(n=42$; 4.70\%), Clinical and Experimental Rheumatology (Clin Exp Rheumatol) ( $n=37 ; 4.13 \%)$, Annals of Rheumatic Diseases (Ann Rheum Dis) $(n=21 ; 2.34 \%)$, and Pediatric Rheumatology (Pediatric Rheumatol) $(n=20 ; 2.23 \%)$. Among the
Egypt, the USA) is presented in green; Cluster 3 (France, Lebanon, the UK) is presented in blue; Cluster 4 (Armenia, Japan) is presented in yellow, and Cluster 5 (Iran, Turkey) is presented in purple

top five journals, Ann Rheum Dis ranked first in total citation count, average citation count and CiteScore 2019 data (Table 3). The cited sources co-citation network visualization map was formed. The full counting method was selected and the minimum number of citations of a journal was set to 150 , which produced 27 journals. The visualization was based on the total number of citations (Fig. 5).

In Vosviewer settings, 12 was entered as the minimum number of occurrences of a keyword and 24 keywords met this requirement. The full counting method was used and the five most frequently used keywords were determined as familial Mediterranean fever (occurrences $=545 ; 40.82 \%$ ), colchicine (occurrences $=97 ; 7.26 \%$ ), fmf (occurrences $=79$; $5.91 \%$ ), amyloidosis (occurrences $=64 ; 4.79 \%$ ), and mefv (occurrences $=60 ; 4.49 \%$ ). The visualization was based on the total number of occurrences and the visualization map is presented in Fig. 6.

Funding sources are listed by the number of studies they supported and the top five were determined to be the Japan Society for the Promotion of Science ( $n=21$; Japan), National Institutes of Health ( $n=8$; USA), National Human Genome Research Institute ( $n=7$; USA), National Institute of Allergy and Infectious Diseases ( $n=7$; USA) and National Institute of Arthritis and Musculoskeletal and Skin Diseases ( $n=5$; USA). 


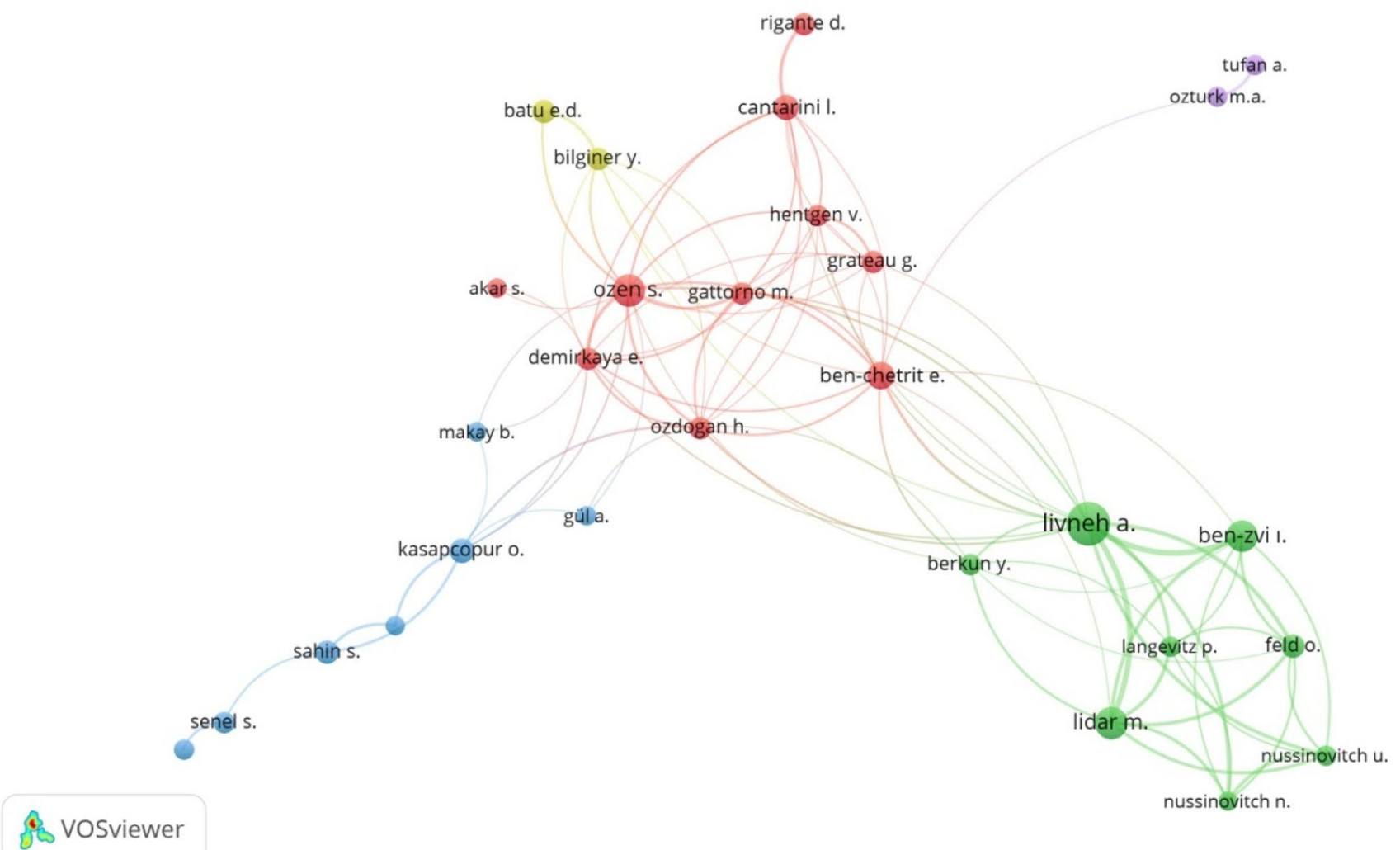

Fig. 4 Author co-authorship network visualization map. Five clusters were created. Cluster 1 (10 authors) is presented in red; Cluster 2 (8 authors) is presented in green; Cluster 3 ( 7 authors) is presented in

blue; Cluster 4 ( 2 authors) is presented in yellow, and Cluster 5 (2 authors) is presented in purple
Table 3 The five most active journals

\begin{tabular}{llccc}
\hline Journal & $n(\%)$ & Total citations & Average citations & CiteScore 2019 \\
\hline Rheumatol Int & $50(5.59)$ & 809 & 16.18 & 3.5 \\
Clin Rheumatol & $42(4.70)$ & 522 & 12.42 & 4.0 \\
Clin Exp Rheumatol & $37(4.13)$ & 334 & 9.02 & 4.5 \\
Ann Rheum Dis & $21(2.34)$ & 1502 & 71.52 & 25.9 \\
Pediatric Rheumatol & $20(2.23)$ & 70 & 3.50 & 4.5 \\
\hline
\end{tabular}

$n$ number of articles, \%: percentage

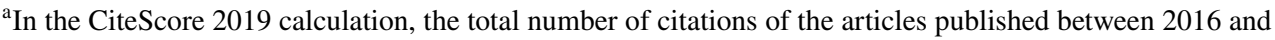
2019 is divided by the total number of articles

\section{Discussion}

Bibliometric analyses present comprehensive data on worldwide scientific productivity, publication trends, the active countries, influential authors and citation values. The results of this study revealed a stable trend in the number of FMF-related articles from 2010 to 2019. This situation can be explained by the low number of main active countries and the considerably low scientific production in this field, except for in particular countries. The fact that the top five countries in terms of the number of articles produced three-quarters of the total articles supports this view.

The top five countries in article production were Turkey, Israel, Japan, Italy and the USA, respectively, and this field was indisputably dominated by Turkey in terms of article production. In the bibliometric study conducted on Behçet, another disease with geographical distribution, it was revealed that Turkey is the dominant country in terms of the number of articles. Consistent with our results, Japan and Italy ranked in the top five [27]. In another FMF-related 
Fig. 5 Cited sources co-citation network visualization map. Three clusters were created. Cluster 1 (14 journals) is presented in red; Cluster 2 (10 journals) is presented in green; Cluster 3 ( 3 journals) is presented in blue

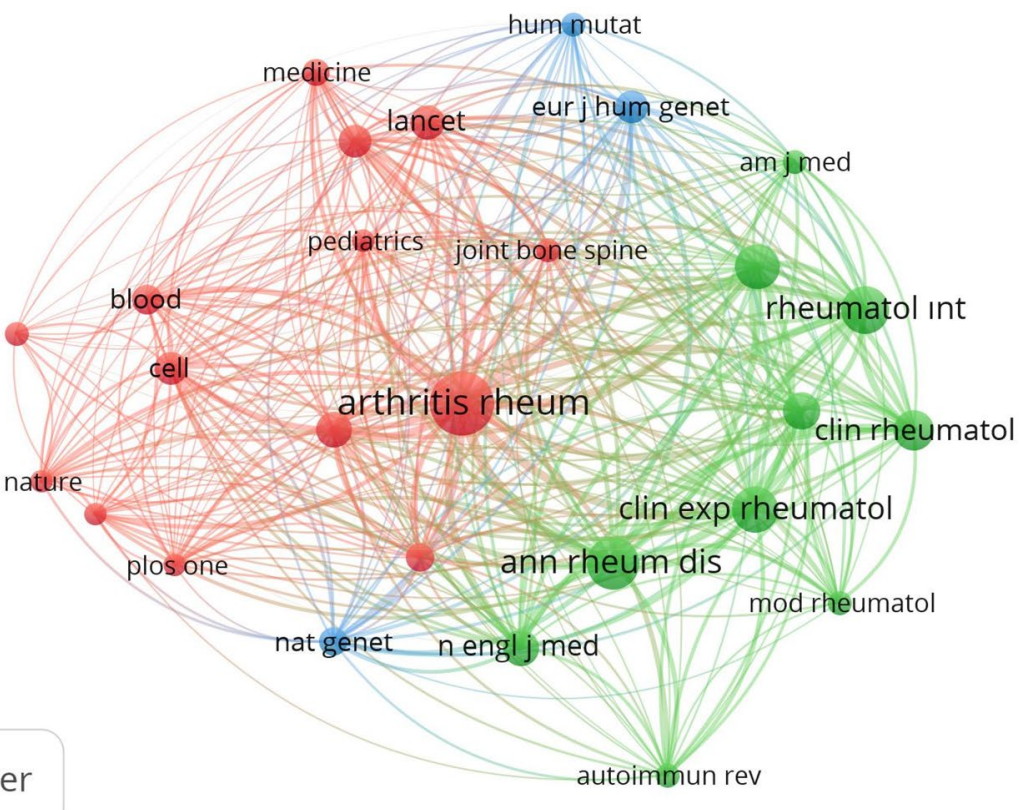

VOSviewer

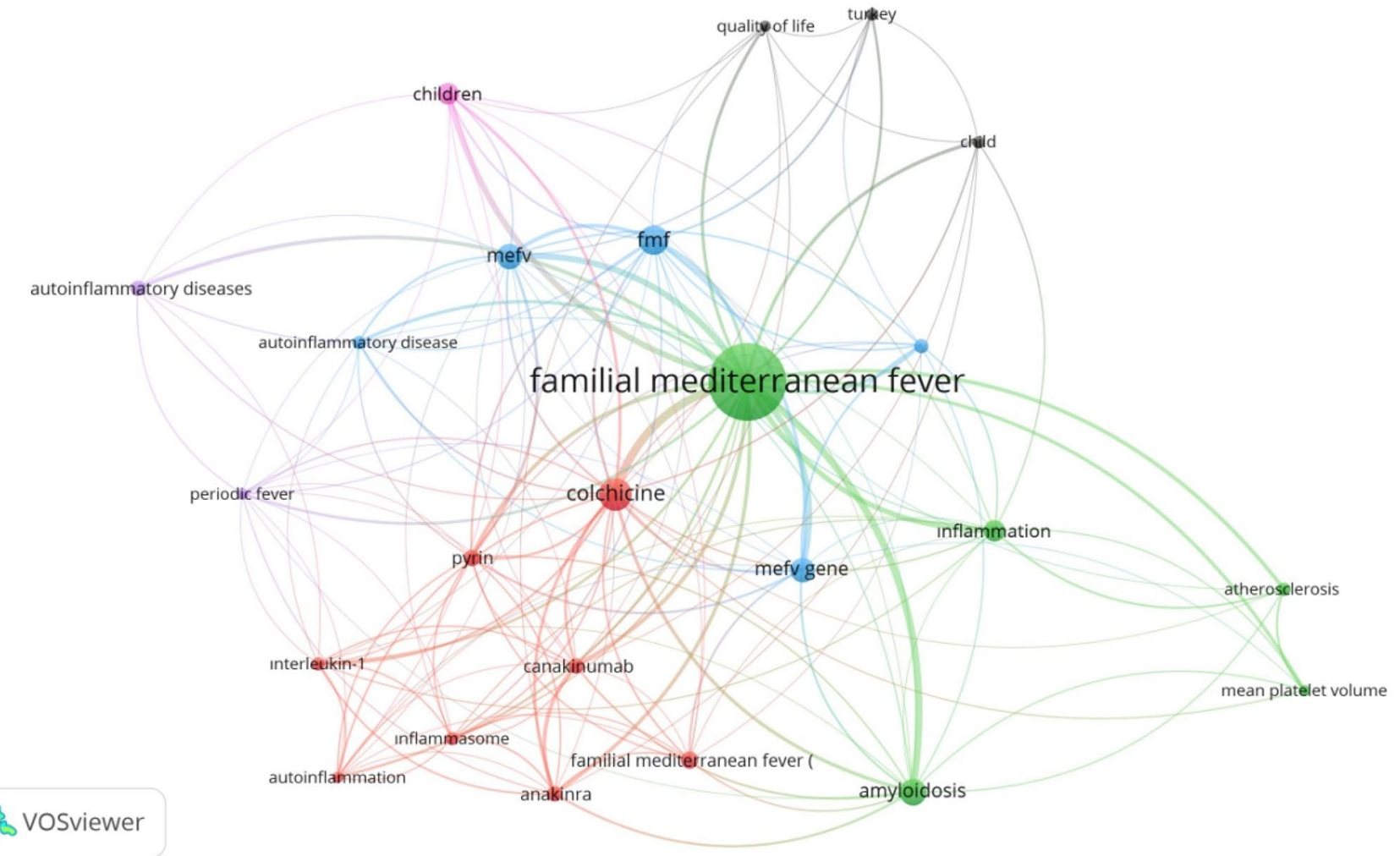

Fig. 6 Author keywords co-occurrence network visualization map. Six clusters were created. Cluster 1 (8 keywords) is presented in red; Cluster 2 (5 keywords) is presented in green; Cluster 3 (5 keywords)

bibliometric study, Turkey was in the first place with a substantial difference in terms of the number of articles [6]. It is not surprising that these countries, where the prevalence of is presented in blue; Cluster 4 (3 keywords) is presented in gray; Cluster 5 (2 keywords) is presented in purple and Cluster 6 (1 keyword) is presented in pink

FMF is high, were in the top five because of the geographical distribution of FMF. Bibliometric studies on Behçet disease also support this view. The high awareness of FMF among 
researchers in these countries may be triggering the production of articles. Despite being outside the geographical distribution, the USAmay have been in the top five as a result of the high budget allocated to scientific research and the presence of reputable researchers from all over the world.

The first three countries in the population-adjusted data were Israel, Armenia and Turkey, and there was seen to be a high number of articles produced by Israel and Armenia compared to their low population sizes. Despite Turkey's high population, the fact that it provided many articles was effective in its entry into the list.

The three leading countries in the number of citations per article were Italy, the USA and France. Yilmaz et al. [12] reported top ten FMF articles according to citation rate and and three articles each from the SA, Israel and France placed in the list. Additionally, only one article from Turkey was available in this list. Although the date range is different, this data shows partial agreement with our results. This result revealed that these three countries, which were also at the forefront in terms of number of articles, do not compromise on quality and reflect their general scientific development in FMF articles.

The five most prolific authors were Ozen S (Turkey), Livneh A (Israel), Lidar M (Israel), Ben-Zvi I ( Israel) and Cantarini L (Italy). In the study, in which the first 100 most cited articles in the field of FMF were evaluated, the first authors of these articles were listed. Livneh A. had seven articles, Ozen S. had three articles, Lidar M. had two articles [12]. These results suggest that the authors who are at the forefront in terms of productivity also provide articles with high scientific impact in the field of FMF. It is expected that the authors of the top five countries in terms of the number of articles will be included in the list. This result suggests that there are scientists who conduct research specifically in the field of FMF in these countries.

The five journals that published the most articles in the field of FMF were Rheumatol Int, Clin Rheumatol, Clin Exp Rheumatol, Ann Rheum Dis and Pediatric Rheumatol, respectively. In the bibliometric study on Behçet disease, Clin Exp Rheumatol (first place), Clin Rheumatol (second place) and Rheumatol Int (fourth place) were among the top five journals [27]. Considering the age group in which FMF emerges, it is an expected result for a pediatric journal to be in the top five. Unlike this, an ophthalmology journal was in the top five in Behçet Disease. The fact that the editors of these journals prioritize FMF articles or that researchers who produce FMF-related articles choose these journals may explain the formation of the specified ranking. Among these journals, Ann Rheum Dis ranked first in total citation count, average citation count and CiteScore 2019 data, indicating that Ann Rheum Dis is not only one of the leading journals in the field of rheumatology, but also highly active in the field of FMF.
The five most frequently used author keywords were familial Mediterranean fever, colchicine, FMF, amyloidosis, and MEFV. It is an expected result that 'familial Mediterranean fever' and 'fmf' would be placed in the list because they are the name and abbreviation of the disease. The fact that 'colchicine', 'amyloidosis' and 'mevf' were in the top five suggests that studies may have focused on FMF treatment, complications, and etiopathogenesis.

The top five funding sources of FMF research were the Japan Society for the Promotion of Science (Japan), National Institutes of Health (USA), National Human Genome Research Institute (USA), National Institute of Allergy and Infectious Diseases (USA) and National Institute of Arthritis and Musculoskeletal and Skin Diseases (USA). In addition to its socioeconomic development, Japan supports research in this area the most because it is a region where FMF is common. This result reveals the importance and support given by Japan to scientific research. The other four funding sources were from the USA. This result explains how the USA is in the top five in terms of article production even though it is not a country in the FMF geography. One of the underlying reasons for the overall scientific development of the USA is its significant support for research.

Conducting the study on a single database can be considered as a limitation. However, this was not considered of great importance as Scopus offers more comprehensive data compared to databases such as Web of Science and Pubmed, which are frequently preferred databases in bibliometric analyses. Scopus has a strong reputation and reliability in the scientific world. Only English articles were evaluated throughout a 10 -year period. The time frame could have been extended, but this date range was set to be able to provide the most up-to-date data.

\section{Conclusion}

Bibliometric analysis is an important data evaluation method in which the global trends of scientific production on specific topics are investigated. The results of this study showed that the number of FMF articles displayed a stable course over the years. To be able to create an upward trend and increase the production of articles, there is a need for researchers in countries other than the main active countries to be supported for FMF articles. Turkey obviously dominated the FMF field in terms of the number of articles, and the USA was the only country in the top five outside of the FMF geography. However, Turkey could not reach a similar ranking in the average number of citations per article. The top three countries in the number of citations per article were Italy, the USA and France. Rheumatol Int was the journal with the highest number of articles published. Among the top five journals, Ann Rheum Dis ranked first in total citation 
count, average citation count, and CiteScore 2019. Given the geographic distribution of FMF, future studies are likely to focus on etiopathogenesis and FMF-related genes. It is obvious that revealing the mechanisms that cause this geographical distribution will have an important place in the future of FMF. Further studies that will enable us to understand the autoinflammatory pathways more clearly may lead to new treatment approaches.

Author contributions BFK designed the study. AA and BFK reviewed the articles and provided the data. BFK analyzed the data. AA contributed the analysis tools. AA and BFK authored and reviewed drafts of the paper. BFK prepared the tables. AA and BFK approved the final manuscript as submitted and agree to be accountable for all aspects of the work.

\section{Declarations}

Conflict of interest The authors declare no conflicts of interest.

Ethical approval No human participants/animals were assessed. These data are public. Therefore, there was no requirement for ethics committee approval.

\section{References}

1. La Regina M, Ben-Chetrit E, Gasparyan AY, Livneh A, Ozdogan H, Manna R (2013) Current trends in colchicine treatment in familial Mediterranean fever. Clin Exp Rheumatol 31:41-46

2. Atas N, Armagan B, Bodakci E, Satis H, Sari A, Bilge NSY, Salman RB, Yardımcı GK, Babaoglu H, Guler AA, Karadeniz H, Kilic L, Ozturk MA, Goker B, Haznedaroglu S, Kalyoncu U, Kasifoglu T, Tufan A (2020) Familial Mediterranean fever is associated with a wide spectrum of inflammatory disorders: results from a large cohort study. Rheumatol Int 40:41-48. https://doi. org/10.1007/s00296-019-04412-7

3. Yüksel S, Ayvazyan L, Gasparyan AY (2010) Familial mediterranean fever as an emerging clinical model of atherogenesis associated with low-grade inflammation. Open Cardiovasc Med J 4:51-56. https://doi.org/10.2174/1874192401004020051

4. Bodur H, Yurdakul FG, Çay HF, Uçar Ü, Keskin Y, Sargın B, Gürer G, Yurdakul OV, Çalış M, Deveci H, Aydın Y, Hizmetli S, Çevik R, Karahan AY, Sunar İ, Duruöz MT, Ecesoy H, Günendi Z, Toprak M, Şen N, Altıntaş D, Cengiz AK, Çă̆layan G, Demir AN, Kaplan H, Ketenci S, Melikoğlu MA, Nayimoğlu M, Nas K, Sarıfakıoğlu AB, Sezer İ (2020) Familial mediterranean fever: assessment of clinical manifestations, pregnancy, genetic mutational analyses, and disease severity in a national cohort. Rheumatol Int 40:29-40. https://doi.org/10.1007/s00296-019-04443-0

5. Sönmez AÖ, Sönmez HE, Çakan M, Yavuz M, Keskindemirci G, Aktay Ayaz N (2020) The evaluation of anxiety, depression and quality of life scores of children and adolescents with familial mediterranean fever. Rheumatol Int 40:757-763. https://doi.org/ 10.1007/s00296-020-04519-2

6. Masri DE, Alsaayed B, Masri JE, Zreika B, Chanbour H, Salameh P (2021) Contribution of Arab countries to familial mediterranean fever research: a pubmed-based bibliometric analysis. Rheumatol Int. https://doi.org/10.1007/s00296-021-04852-0
7. Akca UK, Batu ED (2021) Similarities and differences between familial Mediterranean fever and Bechet's disease. Cent Asian J Med Hypotheses Ethics 2:43-50. https://doi.org/10.47316/ cajmhe.2021.2.1.07

8. Tabatabaei-Malazy O, Atlasi R, Larijani B, Abdollahi M (2016) Trends in publication on evidence-based antioxidative herbal medicines in management of diabetic nephropathy. J Diabetes Metab Disord 15:1. https://doi.org/10.1186/s40200-016-0221-2

9. Kutluk MG, Danis A (2021) Bibliometric analysis of publications on pediatric epilepsy between 1980 and 2018. Childs Nerv Syst 37:617-626. https://doi.org/10.1007/s00381-020-04897-9

10. Bar-Ilan J (2010) Citations to the "Introduction to informetrics" indexed by WOS, Scopus and Google Scholar. Scientometrics 82:495-506. https://doi.org/10.1007/s11192-010-0185-9

11. Tekin AM, Bahşi I (2021) Global research on maxillofacial fracture over the last 40 years: a bibliometric study. J Craniofac Surg. https://doi.org/10.1097/SCS.0000000000007627

12. Yılmaz D, Bektaş Uysal H (2017) Bibliometric analysis of the most cited first one hundred manuscripts in familial mediterranean fever. Meandros Med Dent J 18:96-107. https://doi.org/ $10.4274 /$ meandros. 66375

13. Falagas ME, Pitsouni EI, Malietzis GA, Pappas G (2008) Comparison of PubMed, Scopus, web of science, and Google scholar: strengths and weaknesses. FASEB J 22:338-342. https://doi.org/10.1096/fj.07-9492LSF

14. Mongeon P, Paul-Hus A (2015) The journal coverage of web of science and Scopus: a comparative analysis. Scientometrics 106:213-228. https://doi.org/10.1007/s11192-015-1765-5

15. Sweileh WM, Huijer HA, Al-Jabi SW, Zyoud SH, Sawalha AF (2019) Nursing and midwifery research activity in Arab countries from 1950 to 2017. BMC Health Serv Res 19:340. https:// doi.org/10.1186/s12913-019-4178-y

16. Gasparyan AY, Nurmashev B, Yessirkepov M, Udovik EE, Baryshnikov AA, Kitas GD (2017) The journal impact factor: moving toward an alternative and combined scientometric approach. J Korean Med Sci 32:173-179. https://doi.org/10.3346/jkms. 2017.32.2.173

17. Redondo M, Leon L, Povedano FJ, Abasolo L, Perez-Nieto MA, López-Muñoz F (2017) A bibliometric study of the scientific publications on patient-reported outcomes in rheumatology. Semin Arthritis Rheum 46:828-833. https://doi.org/10.1016/j. semarthrit.2016.12.002

18. Zyoud SH (2019) Global scientific trends on aflatoxin research during 1998-2017: a bibliometric and visualized study. J Occup Med Toxicol 14:27. https://doi.org/10.1186/s12995-019-0248-7

19. Sweileh WM, Sawalha AF, Al-Jabi S, Zyoud SH (2017) Bibliometric analysis of literature on antifungal triazole resistance 1980-2015. Germs 7:19-27. https://doi.org/10.18683/germs. 2017.1104

20. Ekundayo TC, Okoh AI (2018) A global bibliometric analysis of Plesiomonas-related research (1990-2017). PLoS ONE 13:e0207655. https://doi.org/10.1371/journal.pone.0207655

21. Zyoud SH, Waring WS, Al-Jabi SW, Sweileh WM (2017) Global cocaine intoxication research trends during 1975-2015: a bibliometric analysis of web of Science publications. Subst Abuse Treat Prev Policy 12:6. https://doi.org/10.1186/s13011-017-0090-9

22. Akyol A, Kocyigit BF (2021) Publication activity in the field of Sjögren's syndrome: a ten-year Web of Science based analysis. Rheumatol Int 41:763-769. https://doi.org/10.1007/ s00296-020-04714-1

23. Zhao X, Chen J, Pan Y, Feng H, Meng B, Meng Y (2019) A bibliometric analysis of the global research in ankylosing spondyloarthritis (2008-2017). Rheumatol Int 39:1091-1097. https:// doi.org/10.1007/s00296-019-04308-6

24. Zhao X, Ye R, Zhao L, Lin Y, Huang W, He X, Lian F, Tong $X$ (2015) Worldwide research productivity in the field of 
endocrinology and metabolism-a bibliometric analysis. Endokrynol Pol 66:434-442. https://doi.org/10.5603/EP.2015. 0054

25. Akyol A, Kocyigit BF (2021) Ankylosing spondylitis rehabilitation publications and the global productivity: a web of sciencebased bibliometric analysis (2000-2019). Rheumatol Int. https:// doi.org/10.1007/s00296-021-04836-0

26. Van Eck NJ, Waltman L (2010) Software survey: VOSviewer, a computer program for bibliometric mapping. Scientometrics 84:523-538. https://doi.org/10.1007/s11192-009-0146-3
27. Kocyigit BF, Akyol A (2021) Bibliometric and altmetric analyses of publication activity in the field of Behcet's disease in 2010 2019. J Korean Med Sci 36:e207. https://doi.org/10.3346/jkms. 2021.36.e207

Publisher's Note Springer Nature remains neutral with regard to jurisdictional claims in published maps and institutional affiliations. 